\title{
Termografia Infravermelha e Captura de Movimentos: vantagens e desvantagens no desenvolvimento de projetos
}

\author{
Infrared Thermography and Motion Capture: Advantages and Disadvantages in \\ Project Development
}

FORCELINI, Franciele; Mestranda em Design; Universidade Federal de Santa Catarina

francieleforcelini@gmail.com

VARNIER, Thiago; Mestrando em Design; Universidade Federal de Santa Catarina

thiagovarnier1@gmail.com

MERINO, Eugenio Andrés Díaz; Doutor em Eng. Produção; Universidade Federal de Santa Catarina

eugenio.merino@ufsc.br

\begin{abstract}
Resumo
Os instrumentos tecnológicos têm se mostrado como recursos auxiliares na obtenção de dados precisos e confiáveis, porém ainda são utilizados de forma restrita no contexto projetual. Portanto, este artigo tem como objetivo identificar as vantagens e as desvantagens do uso da termografia infravermelha e da captura de movimentos (Xsens) no processo de desenvolvimento de projetos. 0 estudo caracteriza-se como de natureza teórica, com abordagem qualitativa e cunho descritivo. Os resultados apontam que a termografia infravermelha e a captura de movimentos são métodos não invasivos que fornecem múltiplos dados de maneira rápida e confiável. Permitem a obtenção de dados de objetos e/ou sujeitos em movimento nos contextos reais, possibilitando análises imediatas. Assim, auxiliam na deteç̧ão de problemas, identificando fatores de risco, defeitos e falhas de projetos, possibilitando o redesign de produtos, sistemas e serviços. Contudo, são métodos viáveis para o desenvolvimento de projetos mais seguros, confortáveis e eficientes, mantendo o rigor científico.
\end{abstract}

Palavras Chave: termografia infravermelha; captura de movimentos; desenvolvimento de projetos.

\begin{abstract}
Technological instruments have been shown as ancillary resources in obtaining accurate and reliable data, but are still used in a restricted way in the design context. Therefore, this article aims to identify the advantages and disadvantages of using infrared thermography and motion capture (Xsens) in the project development process. The study is characterized as having a theoretical nature, with a qualitative approach and a descriptive character. The results show that infrared thermography and motion capture are non-invasive methods that provide multiple data quickly and reliably. They allow the obtaining of data of objects and / or subjects in movement in the real contexts, enabling immediate analyzes. Thus, they help in the detection of problems, identifying risk factors, defects and project failures, enabling the redesign of products, systems and services. However, they are viable methods for the development of safer, more comfortable and efficient projects, maintaining the scientific rigor.
\end{abstract}

Keywords: infrared thermography; motion capture; project development. 


\section{Introdução}

No processo de desenvolvimento de projetos e de produção de conhecimentos científicos, os dados necessários podem ser obtidos por meio de métodos qualitativos e/ou quantitativos. Os dados qualitativos enfatizam o processo e seu significado, enquanto os quantitativos preocupamse com as medições (TERENCE; ESCRIVÃO FILHO, 2006). São métodos complementares que, quando associados, podem favorecer uma visão mais completa (PASCHOARELLI; MEDOLA; BONFIM, 2015) e uma análise mais objetiva e confiável (MERINO et al., 2017; SPECK et al., 2016).

Os métodos quantitativos visam resultados precisos, a fim de evitar equívocos na análise e interpretação dos dados, gerando maior segurança em relação as informações obtidas. De acordo com Richardson (2008), sua aplicação é frequente em estudos que procuram relações entre variáveis, buscando compreender as características de um fenômeno.

Em relação aos métodos quantitativos de coleta de dados, evidenciam-se os instrumentos tecnológicos, cuja aplicação permite a aferição de dados quantificáveis e precisos (MERINO et al., 2017; SPECK et al., 2016), os quais podem auxiliar em diferentes etapas do projeto. No entanto, no processo de desenvolvimento de projetos em design estes recursos ainda são utilizados de forma restrita. A este respeito, destacam-se diversos instrumentos como a termografia infravermelha, a captura de movimentos, o rastreamento ocular, a eletromiografia de superfície, a dinamometria digital, a goniometria, dentre outros (MERINO et al., 2017; SPECK et al., 2016).

No entanto, este artigo enfatiza a termografia infravermelha e a captura de movimentos. Entende-se a termografia infravermelha como um método de registro das qualidades térmicas superficiais de um corpo (MOBLEY, 2002). Esta tecnologia funciona por meio da captura da energia infravermelha emitida pelos objetos e sujeitos, invisível a olho nú, a qual é convertida em uma imagem térmica (MOBLEY, 2002; SILVA, 2017). A captura de movimentos, por sua vez, caracteriza-se como um método de simulação de movimentos humanos (LÄMKULL et al., 2009). Trata-se de uma técnica utilizada para mapear os movimentos realizados por um objeto e reproduzi-los em ambiente digital (GOMIDE et al., 2009).

Diante do exposto, este artigo visa compreender como a instrumentação tecnológica pode contribuir para o desenvolvimento de projetos. Desta forma, tem como objetivo identificar as vantagens e as desvantagens do uso da termografia infravermelha e da captura de movimentos (Xsens) no processo de desenvolvimento de projetos. Este estudo caracteriza-se como de natureza teórica, abordagem qualitativa e cunho descritivo.

Contudo, este estudo justifica-se por razões teóricas e práticas. A utilização de instrumentos tecnológicos, como a termografia infravermelha e a captura de movimentos, pode possibilitar a obtenção de informações e definições precisas sobre os contextos de estudo, contribuindo para análises mais confiáveis. Estes instrumentos permitem um mapeamento rápido, seguro e preciso (FLIR, 2016; XSENS, 2012).

No que tange às razões práticas, Speck et al. (2016) destaca a importância dos dados quantitativos na concepção e avaliação de novos produtos, tornando-os mais adequados e satisfatórios aos usuários e, consequentemente, reduzindo a necessidade de reprojetos. Em avaliações ergonômicas, Marras et al. (2010) destacam a capacidade da instrumentação em rastrear dados continuamente sem interferir com a rotina de um trabalhador, possibilitando a obtenção de dados confiáveis que descrevem as exposições e os ciclos das operações. 


\section{Fundamentação Teórica}

\subsection{Termografia infravermelha}

A termografia infravermelha é uma técnica baseada na deteç̧ão e registro das qualidades térmicas superficiais de objetos e sujeitos. Essa deteç̧ão acontece por meio de um equipamento que captura a radiação infravermelha e a converte em uma imagem térmica, chamada de termograma, que exprime as variações de temperatura por meio de um espectro de cores, que seriam imperceptíveis a olho nu (CERDEIRA et al., 2011; MOBLEY, 2002; SILVA, 2017). Ou seja, caracteriza-se como uma técnica de imagem que apresenta a distribuição da radiação térmica, transformando-a em valores de temperatura (GABRIEL et al., 2016).

Sua evolução história decorre há mais de dois séculos e encontra-se relacionada às observações feitas pelo médico, filósofo e pesquisador Grego Hipócrates, que identificou variações de temperatura corporais por meio da alteração das características da argila, utilizada como instrumento de avaliação (BRIOSCHI; MACEDO; MACEDO, 2003). Mais tarde, Willians e John Herschel realizaram as primeiras imagens termográficas utilizando o sistema infravermelho por meio da técnica evaporográfica (HOLST, 2000). A figura 1 demonstra uma síntese dos principais eventos relacionados ao desenvolvimento deste método.

Figura 1 - Origem e evolução da Termografia Infravermelha

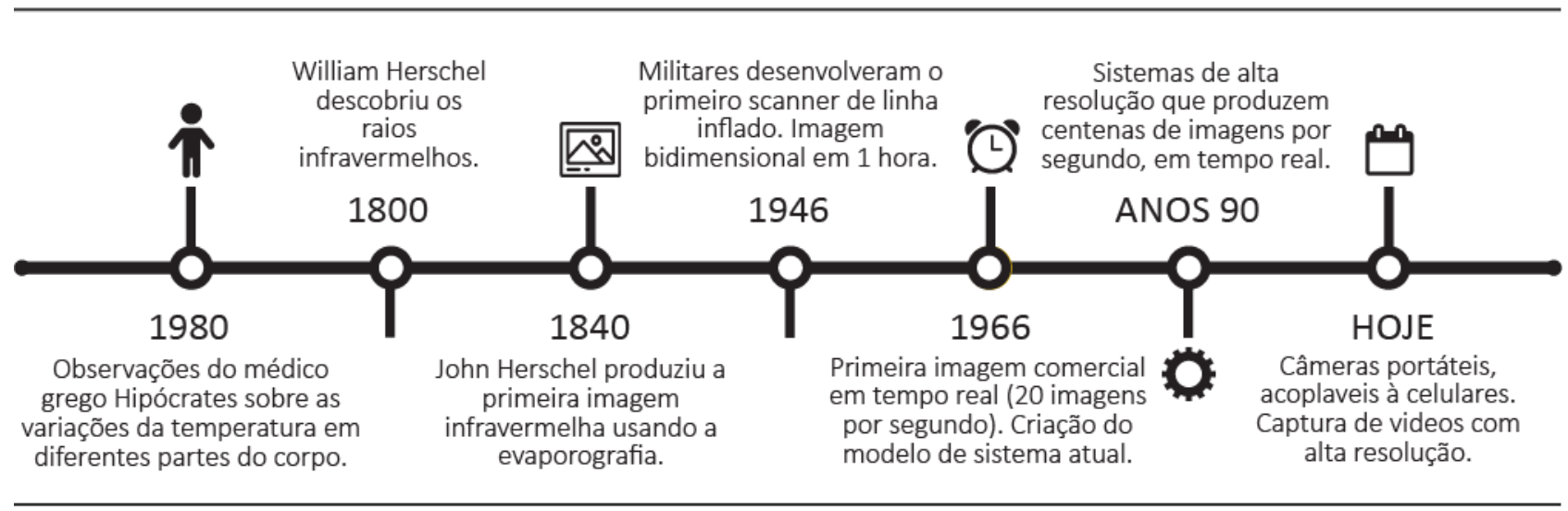

Fonte: Elaborado pelos autores com base em Brioschi, Macedo e Macedo (2003), Holst (2000) e FLIR (2016).

Nesse sentido, a termografia teve seus maiores avanços tecnológicos nas décadas de 1970, 1980 e 1990, com os sistemas de alta resolução, capazes de produzir centenas de imagens por segundo e processar as imagens em tempo real (HOLST, 2000). Hoje, a termografia infravermelha possui aporte de diversos modelos de câmeras térmicas, como as profissionais, compactas e adaptadas à smartphones.

As câmeras termográficas, também chamadas de termovisores, são os instrumentos que permitem a captura das ondas, detectando o infravermelho em uma escala do espectro eletromagnético de aproximadamente 2.000-13,000 nanômetros ou 2-13 $\mu \mathrm{m}$. (FLIR, 2016). Desta forma, os termovisores capturam a energia infravermelha emitida pelos objetos e sujeitos, transformando-a em valores de temperatura e gerando o termograma, conforme demonstrado na figura 2. 
Figura 2 - Processo da captura termográfica

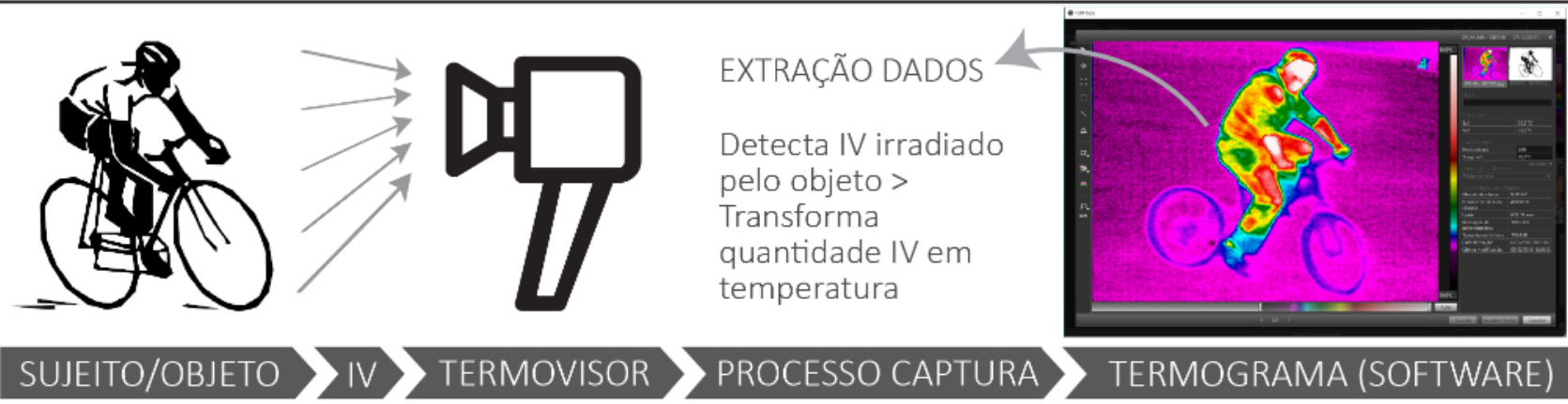

Fonte: Adaptado de SILVA (2017).

O termograma gerado possibilita a visualização de áreas e variações térmicas da superfície em estudo, que podem ser analisadas pelos softwares associados aos termovisores, os quais apresentam ferramentas para análise estatística e visual em tempo real e pós-evento de imagens estáticas e sequências de vídeos (JENKINS, BROWN, RUTTENFORD, 2009).

\subsection{Captura de movimentos}

O sistema de Captura de Movimentos caracteriza-se como uma técnica utilizada para mapear os movimentos realizados por um objeto e reproduzi-los em ambiente digital (GOMIDE et al., 2009). Segundo Streit (2013) é utilizado na biomecânica para registrar o movimento de um corpo por meio de algum dispositivo e, a partir dos dados gerados, calcular as variáveis cinemáticas do movimento.

As discussões sobre a origem da análise biomecânica do movimento humano foram protagonizadas por Aristóteles (370 a.C), seguidas por Leonardo Da Vinci (1452-1519), Giovanni Alfonso Borelli (1608-1679), Eadweard Muybridge (1831-1904), Étienne-Jules Marey (1830-1904), conforme pode ser observado na figura 3, que demonstra uma síntese dos principais eventos relacionados ao desenvolvimento deste método.

Figura 3 - Origem e evolução da Captura de Movimentos

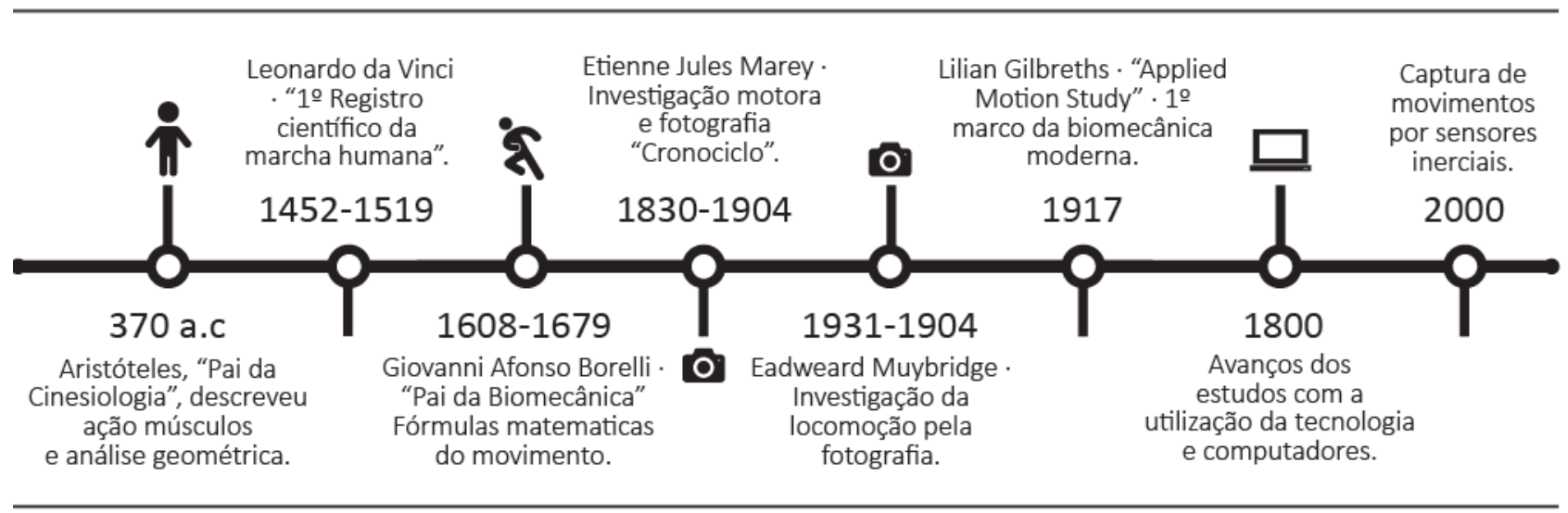

Fonte: Elaborado pelos autores com base em Rasch (1991) e Settineri; Rodrigues (1976).

A evolução da captura de movimentos passou por diversas fases, associadas a diferentes 
técnicas de captura. Iniciou com as técnicas de desenho de Aristóteles, a fotografia em redes lineares de Muybridge, os métodos gráficos e fotográficos de Marey (capazes de capturar e gravar na mesma imagem 12 frames por segundo) e, por fim, a captura de dados por meio de vídeos (RASCH, 1991, SETTINERI; RODRIGUES, 1976).

A partir da década de 1980, com a utilização da tecnologia e dos computadores, os estudos da captura de movimentos puderam evoluir, possibilitando o registro do movimento humano por meio de dispositivos que mensuram as variáveis cinemáticas. Este fato permitiu o aumento da qualidade dos movimentos capturados e o uso de sistemas integrados, reduzindo assim o tempo de processamento, armazenamento e representação da informação (STREIT, 2013). Como exemplo destes dispositivos, tem-se o sistema de captura de movimento 3D Xsens MVN Biomech.

O Xsens é um sistema baseado em unidades de medidas inerciais (IMU's), ou seja, modelos biomecânicos e algoritmos de fusão de sensores que combinam sinais tridimensionais de giroscópio e acelerômetros para rastrear o posicionamento de um objeto, determinando sua velocidade, aceleração e posição (XSENS, 2012).

Esse sistema, conforme demonstra a figura 4, permite a captação de movimentos por meio de 17 módulos de sensores inerciais e magnéticos, gerando 120 frames por segundo $(120 \mathrm{~Hz})$ que possibilitam a avaliação contínua do movimento. Os dados captados são transmitidos via (wireless) para um computador com um software (MVN), permitindo a observação, gravação e análise dos movimentos a partir de gráficos dos ângulos das articulações, da velocidade e duração dos movimentos (SPECK et al., 2016; ROETENBERG; LUINGE; SLYCKE, 2013).

Figura 4 - Posicionamento dos sensores inerciais e interface do software Xsens

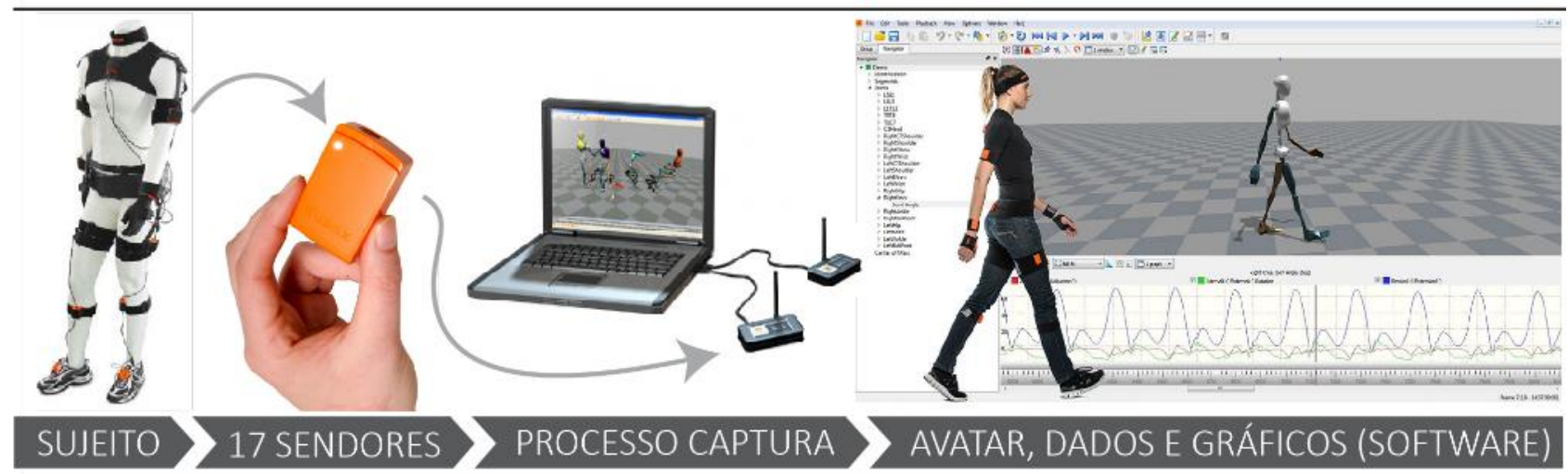

Fonte: Elaborado pelos autores com base em Xsens (2012).

A captura dos movimentos do corpo humano (andar, correr, saltar, rastejar, etc.) pode ser realizada de forma eficaz e em tempo real, possibilitando estudos e avaliações da execução de atividades em seus contextos reais. $O$ sistema pode ser utilizado ao ar livre ou em interiores, visto que não apresenta restrições de iluminação nem constrangimentos associados às oclusões ou falta de marcadores (SPECK et al., 2016; ROETENBERG; LUINGE; SLYCKE, 2013).

\section{Procedimentos Metodológicos}

Este estudo caracteriza-se como teórico, que segundo Marconi e Lakatos (2007) tem por objetivo o progresso científico e a ampliação de conhecimentos teóricos. Dessa forma, o artigo 
visa apresentar vantagens e desvantagens sobre o uso da termografia infravermelha e da captura de movimentos (Xsens) no desenvolvimento de projetos.

Utilizou-se a abordagem qualitativa que, segundo Creswell (2010) visa explorar e entender o significado atribuídos a um problema social ou humano. Deste modo, a pesquisa buscou compreender a termografia infravermelha e a captura de movimentos (Xsens), destacando suas vantagens e desvantagens no desenvolvimento de projetos.

No que tange seus objetivos, classifica-se como descritiva, visto que busca especificar as propriedades, as características, objetos ou outros fenômenos que se submetam a uma análise (SAMPIERI; COLLADO; LUCIO, 2013). Logo, este artigo se propõe a apresentar estudos que utilizaram a termografia infravermelha e a captura de movimentos (Xsens) para a obtenção de dados no processo de desenvolvimento de projeto.

\subsection{Etapas de pesquisa}

A pesquisa segmenta-se em duas etapas: etapa 1 -Mapear; etapa 2 - Analisar e identificar. A primeira etapa (Mapear) contempla a realização da pesquisa bibliográfica, elaborada a partir de materiais já publicados sobre o tema proposto. Na segunda etapa (Analisar e Identificar) foi realizada a análise dos documentos levantados na etapa anterior com o intuito de identificar as vantagens e desvantagens da termografia infravermelha e da captura de movimentos.

\subsection{Materiais e Métodos}

Na etapa 1 (Mapear), foi realizado o levantamento bibliográfico, nos meses de outubro e novembro de 2017. Este levantamento ocorreu de forma assistemática com base em teses e dissertações, bem como artigos de periódicos nacionais e internacionais. Posteriormente, na etapa 2 (Analisar e identificar), os documentos foram selecionados tendo como critérios de inclusão: estudos que abordavam a utilização da instrumentação tecnológica - captura de movimentos e termografia infravermelha. Após esta seleção, foi realizado a extração dos dados, por meio de tabelas, identificando as vantagens e desvantagens da instrumentação tecnológica. Por fim, foi realizado a organização e apresentação dos resultados.

\section{Resultados}

Neste tópico são apresentadas as vantagens e as desvantagens da aplicação da termografia infravermelha e da captura de movimentos (Xsens), a fim de se compreender como a instrumentação tecnológica pode contribuir para o desenvolvimento de projetos.

\subsection{Termografia infravermelha}

A termografia infravermelha destaca-se pela capacidade de exploração aprofundada e menos subjetiva dos sujeitos e objetos de estudo, possibilitando a identificação de um quadro visual térmico que permite a comparação de temperaturas (SILVA; TARALLI; MELZ, 2015). Assim, a técnica, de caráter bidimensional, pode ser utilizada na medição de temperatura da superfície de todos os tipos de materiais (GIORLEO; MEOLA, 2002).

Caracteriza-se como um método de medição não destrutivo, passivo e sem contato direto, que permite o mapeamento térmico de um corpo por meio da radiação infravermelha emitida pelas superfícies (BRIOSCHI; MACEDO; MACEDO, 2003; GABRIEL et al., 2016; HOLST, 2000; SILVA; TARALLI; MELZ, 2015). Captura a radiação (não-ionizante) naturalmente emitida pelo corpo humano, não causando qualquer perigo (GABRIEL et al., 2016). 
A termografia infravermelha possui aporte de diversos modelos de câmeras térmicas, o que possibilita maior acessibilidade e visibilidade a técnica. As câmeras termográficas modernas possuem versões acessíveis, portáteis (versões de mão) e potentes, com altos níveis de sensibilidade térmica e resolução espacial (JENKINS; BROWN, RUTTENFORD, 2009).

De acordo com Silva, Taralli e Melz (2015), a técnica possui compatibilidade com diversos softwares para a interpretação das imagens térmicas. Os pacotes de softwares, normalmente associados às câmeras térmicas, apresentam uma variedade de ferramentas avançadas para análise estatística e visual em tempo real e após a captura de imagens estáticas e sequências de vídeos (JENKINS, BROWN, RUTTENFORD, 2009).

Por meio da termografia infravermelha, torna-se possível observar sujeitos e objetos de maneira confiável em situações diversas. A este respeito, Merino et al. (2017) e Speck et al. (2016) destacam a possibilidade da obtenção de dados precisos dos sujeitos, mesmo quando apresentam limitações físicas e/ou psíquicas que dificultam ou impedem sua comunicação e, consequentemente, o acesso às informações necessárias ao projeto. Deste modo, é possível manter o rigor científico e ainda respeitar capacidades e limitações dos sujeitos envolvidos.

Essa técnica destaca-se pela sua rapidez, reprodutibilidade e, quando comparada com outras modalidades, pelo baixo custo (GABRIEL et al., 2016). Nos setores industriais, Silva (2017) destaca a possibilidade da detecção rápida de problemas, do aumento dos rendimentos e segurança dos sistemas, da redução dos custos de manutenção, custos operacionais e associados à imprevistos.

A termografia infravermelha não interfere nos processos e sistemas de trabalho dos objetos analisados, possibilitando uma diversidade de aplicações. Para Silva, Taralli e Melz (2015) suas aplicações podem auxiliar no diagnóstico do mau uso de materiais e peças, de defeitos de materiais, de falhas de projeto, bem como pode ser associado a técnicas subjetivas para avaliar a percepção dos usuários, proporcionando o redesign de produtos. Silva (2017) destaca que, por meio deste método, pode-se capturar e analisar objetos em movimento, bem como avaliar padrões térmicos por meio da análise de uma imagem. Ainda, ressalta que, rapidamente, podem ser coletados múltiplos dados térmicos.

Quanto às desvantagens da termografia infravermelha, Silva, Taralli e Melz (2015) destacam a limitação da deteç̧ão de apenas temperaturas superficiais dos objetos e sujeitos, as possíveis interferências das condições do ambiente nas medições térmicas, bem como a dificuldade na interpretação das imagens de determinados materiais devido sua emissividade. Além disso, também são identificadas como desvantagens a precisão limitada de algumas câmeras termográficas e os valores elevados das câmeras de alta qualidade (SILVA, 2017; SILVA; TARALLI; MELZ, 2015). Não obstante, Silva (2017) destaca a necessidade do envolvimento de profissionais capacitados no levantamento de dados com o equipamento, com conhecimentos técnicos sobre o uso desta tecnologia.

\subsection{Captura de movimento (Xsens)}

A captura de movimentos (Xsens) é salientada nos estudos de Saber-Sheikh et al. (2010), Dinu et al. (2012) e Zhang et al. (2013) como um método propício para avaliar os parâmetros da cinemática 3D da marcha, devido sua precisão e confiabilidade durante o registro das informações. Zhang et al. (2013) destaca a precisão dos dados de flexão e extensão (plano sagital) das articulações do quadril, joelho e tornozelo. Paiva (2016) ressalta sua capacidade de recolher 
dados cinemáticos no plano sagital das articulações do membro inferior, mostrando-se sensível para detectar alterações nos parâmetros angulares da marcha da população idosa.

A integração contínua dos sinais do acelerômetro e do giroscópio atualizam o modelo biomecânico com taxa máxima de $120 \mathrm{~Hz}$, o que permite uma avaliação contínua do movimento (SABER-SHEIKH et al.,2010; DINU et al., 2012; ZHANG et al., 2013). Nesse sentido, Sato, Usui e Watabe (2012) destacam a aplicação da captura de movimentos no ensino da dança, tornando possível mostrar os movimentos aos alunos, esclarecendo pontos a serem corrigidos.

A captura de movimentos (Xsens) é abordada por Cunha et al. (2015) devido sua capacidade de identificar com clareza os fatores de risco referentes às atividades, além de gerar dados precisos sobre a biomecânica. Portanto, vem sendo utilizada em diversos setores, como de entretenimento, pesquisas médicas, esportivas, militares, projetos ergonômicos, avaliação de ambientes de trabalho, simulações virtuais, bem como o desenvolvimento de produtos (STREIT, 2013). Além disso, foi introduzida na indústria como uma ferramenta para facilitar processos de projetos, acelerando seu desenvolvimento e eficiência em custos (LÄMKULL et al., 2009).

Este método é destacado por Gandy et al. (2014) como uma técnica não invasiva, eficiente e prática, capaz de registrar dados precisos. Em uma análise das interações entre cavalos e cavaleiros, os autores verificaram que a captura de movimentos permitiu o registro preciso da assimetria da rotação do quadril.

O sistema inercial do Xsens não requer um sistema de câmeras externas, emissores ou marcadores para obtenção dos dados, permitindo o estudo do movimento humano fora do contexto laboratorial (STREIT, 2013). Logo, pode ser utilizado ao ar livre e/ou em interiores (habitação, laboratórios), visto que não apresenta restrições de iluminação e constrangimentos associados às oclusões ou à falta de marcadores (ROETENBERG; LUINGE; SLYCKE, 2013).

A este respeito, Paiva (2016) enfatiza que o sistema de sensores inerciais se mostra adequados para a captura de dados da cinemática angular fora do contexto laboratorial. Silva et al. (2014) destacam o Xsens como adequado para a avaliação do trabalho odontológico, oferecendo dados cinemáticos confiáveis e limpos. Além disso, salienta que os sensores inerciais de movimento fornecem medidas precisas de ângulo de cabeça e ombros, quando comparadas a outros estudos usando inclinômetros e goniômetros.

Quanto às desvantagens da captura de movimentos, Salvalaio (2012) aborda que os sensores inerciais têm demonstrado inconvenientes quanto a quantidade de fios presos ao sensor em cada articulação. Ainda, destaca a dificuldade no ajuste dos sensores sobre o corpo, justificando que a precisão da captura do movimento depende do exato posicionamento sobre o vértice das articulações.

No que se refere aos sensores inerciais, Carvalho (2011) aborda que estes estão sujeitos a desvios, que diminuem a qualidade da captura, acarretam erros nos dados e, consequentemente, diminuem a acurácia dos movimentos capturados em relação aos reais. $O$ autor justifica esse fato pelas imprecisões do fator de escala, desalinhamento e ruído, que evoluem com o tempo de captura e são intrínsecos ao processo. Para minimizar esses erros, recomenda-se a utilização dos magnetômetros e a redução do ciclo de captura das gravações, o que garante maior confiabilidade nos dados (CARVALHO, 2011; SABER-SHEIKH et al., 2010; DINU et al., 2012; ZHANG et al., 2013). 
O Xsens apresenta limitações em relação aos dados nos planos coronal e transversal do movimento, justificando o uso no plano sagital, que revela níveis elevados de validade (NOVAK et al., 2014; ZHANG et al., 2013; ROETENBERG; LUINGE; SLYCKE, 2013). No entanto, para Novak et al. (2014) e Weenk et al. (2013), o sistema é satisfatório quando comparado com a maioria dos algoritmos de análise de marcha. Por fim, Streit (2013) relata o sistema como dispendioso em relação ao custo.

\subsection{Síntese dos resultados}

Diante dos resultados apresentados, a figura 5 apresenta uma síntese das vantagens e desvantagens da termografia infravermelha e da captura de movimentos, bem como as vantagens e desvantagens compartilhadas por ambas as tecnologias.

Figura 5 - Síntese das vantagens e desvantagens da termografia infravermelha e da captura de movimentos
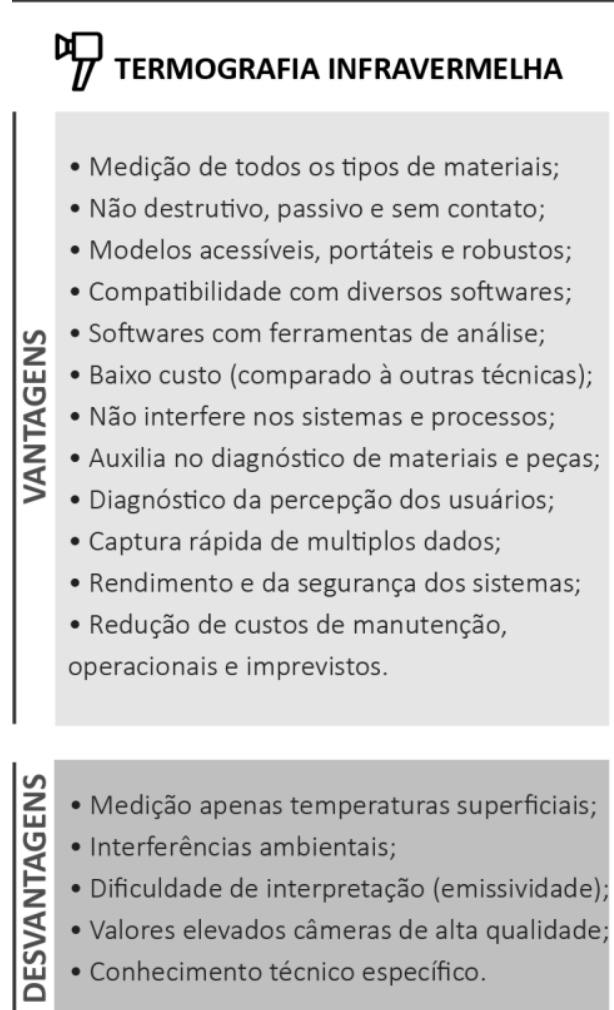

\section{骂}

- Exploração aprofundada;

- Dados objetivos e precisos;

- Análise em tempo real ou após captura;

- Captura em situações diversas;

- Coletas com sujeitos com limitações;

- Rapidez e reprodutibilidade;

- Detecção de problemas;

- Auxilia no desenvolvimento de produtos;

- Captura/análise objetos em movimento:

- Auxilia em projetos ergonômicos;

- Facila processos de projetos;

\section{CAPTURA DE MOVIMENTO (XSENS)}

- Método propício para análise da marcha; - Precisão dos dados de flexão e extensão

(plano sagital) - quadril, joelho e tornozelo; - Avaliação contínua do movimento;

- Identificação de fatores de risco;

- Dados precisos da biomecânica;

- Auxilia avaliação ambientes de trabalho;

- Técnica não invasiva, eficiente e prática;

- Estudo fora do contexto laboratorial;

- Angulos precisos (cabeça e ombros);

- Não possui restriçães de iluminação e contrangimentos pela falta de marcadores.

\section{- Não apresentam}

desvantagens similares.
- Quantidade de fios presos ao sensores;

- Dificuldade de ajuste dos sensores;

- Sensores sujeitos à desvios;

- Limitações planos coronal e transversal;

- Dispendioso em relação ao custo.

Fonte: Elaborado pelos autores.

No desenvolvimento de projetos, a termografia infravermelha e a captura de movimentos podem contribuir de maneiras diversas, conforme pode ser observado na figura 6 . Estes instrumentos possibilitam coletas não invasivas de maneira rápida e confiável, respeitando as capacidades e limitações do usuário, gerando múltiplos dados que podem subsidiar as diferentes etapas de projetos. Não obstante, permitem a obtenção de dados de objetos e/ou sujeitos em movimento nos contextos reais, possibilitando análises imediatas. 
Figura 6 - Contribuições da termografia infravermelha e da captura de movimentos no desenvolvimento de projetos

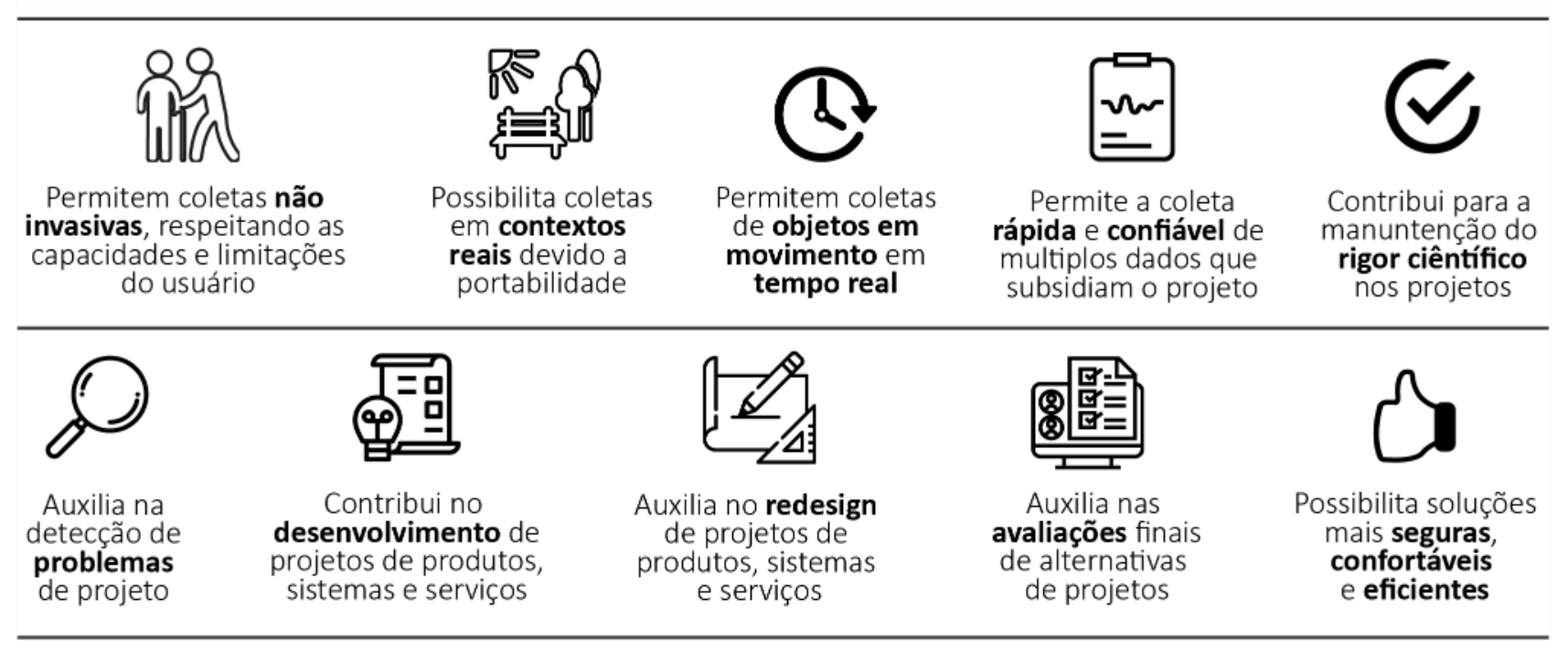

Fonte: Elaborado pelos autores.

Nas etapas iniciais do projeto, a termografia infravermelha e a captura de movimentos podem auxiliar na detecção de problemas, identificação de fatores de risco, bem como de defeitos e falhas de projetos. Dessa forma, auxiliam no redesign de produtos, sistemas e serviços e na avaliação de alternativas de projetos. Contudo, essas tecnologias contribuem para o desenvolvimento de soluções mais seguras, confortáveis e eficientes, mantendo o rigor científico devido a confiabilidade dos dados.

\section{Conclusão}

Diante dos resultados, entende-se que a termografia infravermelha e a captura de movimentos podem ser utilizadas para ampliar a visibilidade dos fenômenos, visto que permitem uma exploração aprofundada dos sujeitos e objetos de estudo. Assim, podem contribuir para o processo de desenvolvimento de projetos, auxiliando na obtenção de medições objetivas (quantificáveis), gerando informações e definições precisas.

Estes instrumentos permitem a aferição de dados quantificáveis que podem ser analisados em tempo real ou após a captura. Possibilitam rapidez e reprodutibilidade nas coletas, podendo ser usados na detecção de problemas, análises ergonômicas, bem como no desenvolvimento de novos projetos ou adaptações de produtos existentes. Além disso, se mostram adequados para coletas envolvendo sujeitos com limitações, mantendo o rigor científico sem desconsiderar as necessidades do usuário e do projeto.

São tecnologias que se destacam pela portabilidade, o que permite maior flexibilidade nas aferições de dados e facilita o processo de projeto. Portanto, são utilizados em diferentes áreas de conhecimento, como a medicina, veterinária, engenharia, design, ergonomia, entre outras.

A termografia infravermelha possibilita uma captura rápida de múltiplos dados e pode ser utilizada em projetos sem interferir nos sujeitos, objetos, sistemas e processos analisados. Desta forma, pode auxiliar em diagnósticos diversos. Na indústria, pode propiciar o aumento do rendimento e da segurança dos sistemas, bem como a redução de custos.

A tecnologia da captura de movimento, por sua vez, permite aos pesquisadores uma mensuração precisa da biomecânica por meio da avaliação contínua do movimento, o que 
possibilita a identificação dos fatores de risco associados aos sujeitos. Os dados gerados facilitam o processo de projeto, auxiliando no desenvolvimento e avaliação de produtos e atividades, bem como na análise das interações entre usuário e objeto.

Este artigo analisou e identificou vantagens e desvantagens da aplicação da termografia infravermelha e da captura de movimentos em estudos de áreas e abordagens diversas, no entanto, propõe-se novas pesquisas relacionadas a áreas específicas como a de tecnologia assistiva, ergonomia, usabilidade, entre outras. Outro tópico a ser analisado refere-se aos protocolos utilizados para a coleta de dados com o uso da instrumentação tecnológica.

\section{Referências}

BRIOSCHI, Marcos L.; MACEDO, José F; MACEDO, Rodrigo de A. C. Termometria cutânea: novos conceitos. Jornal Vascular Brasileiro, v. 2, p. 151-60, 2003.

CARVALHO, Adriano G. Influência da modelagem dos componentes de bias instabilidade dos sensores inerciais no desempenho do navegador integrado SNI/GPS. Instituto Militar de Engenharia, Rio de Janeiro. 2011.

CERDEIRA, Fernando et al. Applicability of infrared thermography to the study of the behaviour of stone panels as building envelopes. Energy and Buildings, v. 43, n. 8, p. 1845-1851, 2011.

CRESWELL, John W. Projeto de pesquisa: Métodos qualitativo, quantitativo e misto. 3 ed. Porto Alegre: Artmed, 2010.

CUNHA, Julia $M$. et al. O uso de sensores inerciais (xsens) no projeto de produto aplicado a ferramenta agrícola. Anais de congresso. Fourth International Conference on Integration of Design, Engineering and Management for innovation, Florianópolis/ SC, Brazil, 2015.

DINU, Daniel et al. Accuracy of Postural Human-motion Tracking Using Miniature Inertial Sensors. Procedia Engineering, v. 147, p. 655-658, 2016.

FLIR. Manual do utilizador: Série FLIR Exx. (2016). Disponível em: <http://support.flir.com>. Acesso em: 25 set. 2017.

GABRIEL, Joaquim et al. Termografia: imagem médica e síndromes dolorosas. Lisboa: Lidel, 2016.

GANDY, Elizabeth A. et al. A preliminary investigation of the use of inertial sensing technology for the measurement of hip rotation asymmetry in horse riders. Sports Technology, v. 7, n. 1-2, p. 7988, 2014.

GIORLEO, Giuseppe; MEOLA, Carosena. Comparison between pulsed and modulated thermography in glass-epoxy laminates. Ndt \& e International, London, v. 35, n. 5, p. 287-292, jul. 2002. Elsevier BV. http://dx.doi.org/10.1016/s0963-8695(01)00062-7.

GOMIDE, João Victor B. et al. Captura de movimento e Animação de Personagens em Jogos. In: Brazilian Symposium on Games and Digital Entertainment, 8., 2009, Rio de Janeiro. Simpósio... Rio de Janeiro: PUC, 2009.

HOLST, Gerald C. Common sense approach to thermal imaging. Washington, DC, USA: SPIE Optical Engineering Press, 2000. 
JENKINS, Sean, BROWN, Raymond; RUTTENFORD, Nail. Comparing thermographic, EEG, and subjective measures of affective experience during simulated product interactions. International Journal of Design. Taipei, v. 3, n. 2, p.53-65, ago. 2009.

LÄMKULL, Dan; HANSON, Lars; ÖRTENGREN, Roland. A comparative study of digital human modelling simulation results and their outcomes in reality: A case study within manual assembly of automobiles. International Journal of Industrial Ergonomics, v. 39, n. 2, p. 428-441, 2009.

MARCONI, Marina de A.; LAKATOS, Eva Maria. Técnicas de pesquisa: planejamento e execução de pesquisas, amostragens e técnicas de pesquisas, elaboração, análise e interpretação de dados. 6.ed. São Paulo: Atlas, 2007.

MARRAS, William S. et al. Instrumentation for measuring dynamic spinal load moment exposures in the workplace. Journal Of Electromyography And Kinesiology, [s.I.], v. 20, n. 1, p.1-9, fev. 2010. Elsevier BV. http://dx.doi.org/10.1016/j.jelekin.2008.12.001.

MERINO, Eugenio et al. Implementation of Integrated Instrumentation in Assistive Technology. Advances in Ergonomics in Design, [s.l.], p.549-560, 24 jun. 2017. Springer International Publishing. http://dx.doi.org/10.1007/978-3-319-60582-1_55.

MOBLEY, R. Keith. An introduction to predictive maintenance. 1. ed. Amsterdam: ButterworthHeinemann, 2002.

NOVAK, Domen et al. Toward real-time automated detection of turns during gait using wearable inertial measurement units. Sensors, v. 14, n. 10, p. 18800-18822, 2014.

PAIVA, Cláudia. Efeito da introdução de tarefas cognitivas nos parâmetros angulares da marcha da população idosa, medido por um sistema ambulatório de análise cinemática tridimensional. 2016. Dissertação (Mestrado) - Curso de Pós-graduação em Fisioterapia, Universidade Nova de Lisboa, Lisboa, 2016. Disponível em: <https://comum.rcaap.pt/handle/10400.26/17791> . Acesso em: 15 jan.2018.

PASCHOARELLI, Luis C.; MEDOLA, Fausto O.; BONFIM, Gabriel H. C. Característica Qualitativas, Quantitativas e Quali-quantitativas de Abordagens Ergonômicas: estudos de caso na subárea do Design Ergonômico. Revista de Design, Tecnologia e Sociedade, v. 2, p. 65-78, 2015.

RASCH, Philip J. Cinesiologia e anatomia aplicada. 7. ed. Rio de Janeiro: Guanabara Koogan, 1991. RICHARDSON, Roberto J. Pesquisa Social: Métodos e Técnicas. 3ạ edição. São Paulo, Atlas, 2008.

ROETENBERG, Daniel; LUINGE, Henk; SLYCKE, Per. Xsens MVN: full 6DOF human motion tracking using miniature inertial sensors. Xsens Motion Technologies BV, Tech. Rep, 2013.

SABER-SHEIKH, Kambiz et al. Feasibility of using inertial sensors to assess human movement. Manual Therapy, v. 15, n. 1, p. 122-125, 2010.

SALVALAIO, Cláudio Luiz. Contribuição ao estudo da captura do movimento aplicado ao design em tecnologia assistiva. 2012. 122 f. Dissertação (Mestrado) - Curso de Pós-design, Engenharia de Materiais, Universidade Federal do Rio Grande do Sul, Porto Alegre, 2012. Disponível em: <http://www.lume.ufrgs.br/handle/10183/62049>. Acesso em: 06 jan. 2018.

SAMPIERI, Roberto H.; COLLADO, Carlos F.; LUCIO, María del P. B. Metodologia de pesquisa. 5.ed. Porto Alegre: Penso, 2013. 
SATO, Katsumi; USUI, Yoko; WATABE, Shinichi. The value to dance practice of CG derived from motion capture. In: SIGGRAPH Asia 2012 Posters. ACM, p. 2, 2012.

SETTINERI, Luiz I. C.; RODRIGUES, Raul B. Fundamentos de cinesiologia. Porto Alegre: Movimento/Universidade Federal do Rio Grande do Sul, 1976.

SILVA, Júlio Cézar R. P. da; TARALLI, Cibele H.; MELZ, Simone P. M. Termograma: A imagem térmica como instrumento de diagnóstico rápido no design. In: Fourth International Conference on Integration of Design, Engineering and Management for innovation. Anais... Florianópolis, SC, Brasil: 2015. Disponível em: <http://janainaramos.com.br/idemi2015/anais/02/143332.pdf>. Acesso em: 10 jan. 2018.

SILVA, Luiz A. da. Termografia: Princípios básicos e suas aplicações. 1. ed. São José dos Campos: Tecnolass Tecnologia, 2017.

SILVA, Vanessa G. da et al. Preliminary inertial study of body postures during dental procedures. Biodental Engineering III, p. 287, 2014.

SPECK, Giselle M. et al. Processo de instrumentação integrada no desenvolvimento de projetos de Tecnologia Assistiva. Anais de congresso. 18 Congresso Brasileiro de Ergonomia (ABERGO), Belo Horizonte: 2016.

STREIT, Priscilla. Comparação de parâmetros biomecânicos entre sistemas de captura de movimentos: avaliação do Microsoft Kinect. 2013. 98 f. Dissertação (Mestrado) - Curso Pósgraduação em Design, da Universidade do Estado do Rio de Janeiro, Rio de Janeiro, 2013. Disponível em: http://bdtd.ibict.br/vufind/Record/UERJ_c6d8f4f128ed6ba8dc3e8174e0d59c26 >. Acesso em: 06 jan.2018.

TERENCE, Ana Cláudia F.; ESCRIVÃO FILHO, Edmundo. Abordagem quantitativa, qualitativa e a utilização da pesquisa-ação nos estudos organizacionais. Encontro Nacional de Engenharia de Produção, v. 26, p. 1-9, 2006.

WEENK, Dirk et al. Automatic identification of inertial sensor placement on human body segments during walking. Journal of neuroengineering and rehabilitatio, $n$, v. 10, n. 1, p. 31, 2013.

XSENS. Moven: user manual. Moven Motion Capture System. The Netherlands: Xsens Technologies B.V. 2012.

ZHANG, Jun-Tian et al. Concurrent validation of Xsens MVN measurement of lower limb joint angular kinematics. Physiological measurement, v. 34, n. 8, p. N63, 2013.

\section{Agradecimentos}

Agradecemos ao Programa de Pós-graduação em Design da UFSC (PPGD/UFSC), ao Núcleo de Gestão de Design e Laboratório de Design e Usabilidade (NGD-LDU/UFSC), à Rede de Pesquisa e Desenvolvimento em Tecnologia Assistiva (RPDTA), à Coordenação de Aperfeiçoamento de Pessoal de Nível Superior (CAPES), ao Conselho Nacional de Desenvolvimento Científico e Tecnológico (CNPq), e aos demais envolvidos. 